\title{
Enhanced optical cooling system test in an electron storage ring
}

\author{
E. G. Bessonov and M. V. Gorbunkov \\ Lebedev Physical Institute, RAS, Moscow, Russia
}

A. A. Mikhailichenko

Cornell University, Ithaca, New York 14853, USA

(Received 18 May 2007; published 18 January 2008)

\begin{abstract}
We are proposing an experiment to test the new idea of enhanced optical cooling (EOC) in an electron storage ring. This experiment will confirm new fundamental processes in beam physics and will demonstrate new unique possibilities with this cooling technique. It will open important applications of EOC in nuclear physics, elementary particle physics, and in light sources based on high brightness electron and ion beams.
\end{abstract}

DOI: 10.1103/PhysRevSTAB.11.011302

PACS numbers: 42.65.Lm, 41.75.Ak

\section{INTRODUCTION}

Emittance and the number of stored particles $-N$ in the beam determine the principal parameter of the beam, its brightness can be defined as $B=N / \gamma \varepsilon_{x} \gamma \varepsilon_{z} \gamma \varepsilon_{s}$, where each $\gamma \varepsilon_{x, z, s}$ stands for invariant emittance associated with the corresponding coordinate. Beam cooling reduces the beam emittance, size, and energy spread in a storage ring and therefore improves its quality for experiments. All high-energy colliders and high-brilliance light sources require intense cooling to reach extreme parameters. Several methods for particle beam cooling are in hand now: (i) radiation cooling, (ii) electron cooling, (iii) stochastic cooling, (iv) optical stochastic cooling, (v) laser cooling, (vi) ionization cooling, and (vii) radiative (stimulated radiation) ion cooling [1-3]. Recently a new method of enhanced optical cooling (EOC) was suggested [4-7]. In this proposal we discuss an experiment which might test the last method in an existing electron storage ring having a maximal energy $\sim 2.5 \mathrm{GeV}$, and which can also function down to energies of $\sim 100-200 \mathrm{MeV}$.

EOC [4] appeared as the symbiosis of enhanced emittance exchange and optical stochastic cooling (OSC) [810]. These ideas have not yet been demonstrated. At the same time the ordinary stochastic cooling (SC) is widely in use in proton and ion colliders. OSC and EOC extend the potential for fast cooling due to bandwidth. EOC can be successfully used in a large hadron collider (LHC) as well as in a planned muon collider.

In the simplest case, the EOC uses one pickup and one or more kicker undulators located at a distance determined by the betatron phase advance $\psi_{x}^{\text {bet }}=2 \pi\left(k_{p, k}+1 / 2\right)$ for first kicker undulator and $\psi_{x}^{\text {bet }}=2 \pi k_{k, k}$ for the next ones, where $k_{i j}=0,1,2,3, \ldots$ are the whole numbers. Other elements of the cooling system are the optical amplifier (typically optical parametric amplifier, i.e., OPA), optical filters, optical lenses, a movable screen, and an optical line with a variable time delay. In this case cooling is produced in the longitudinal and transverse radial phase space.
Undulator radiation (UR) is emitted by a particle in the pickup undulator in the form of undulator radiation wavelets (URWs). They are passed through an optical system with a movable screen located on the image plane of the particle beam. First, the screen in the optical system opens the way for URWs emitted in the pickup undulator by particles with higher deviations of their closed orbits $x_{\eta}$ (higher energies) and higher positive deviations $x_{\beta}>0$ from their closed orbits. The radiation is then amplified and passes through the following kicker undulator(s) together with the particle (see Fig. 1).

If the betatron phase advance for the lattice segment between pickup and kicker undulators is $(2 p+1) \pi$ and the deviation of the particle in the pickup undulator $x_{\beta}>0$,
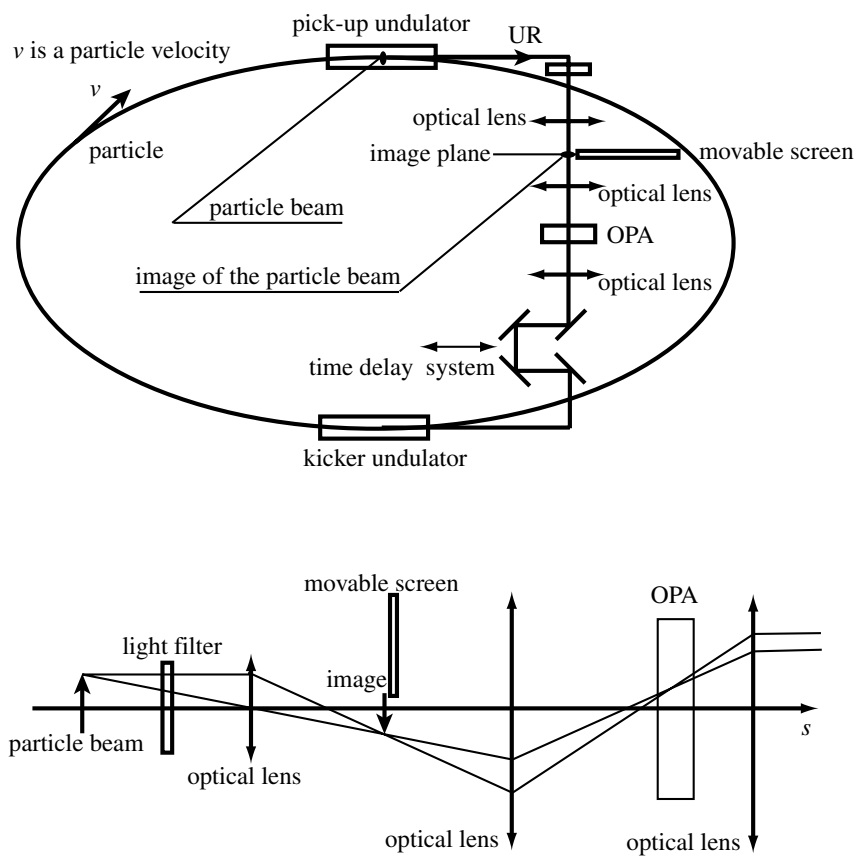

FIG. 1. The scheme of the EOC of a particle beam (a) and unwrapped optical scheme (b). 


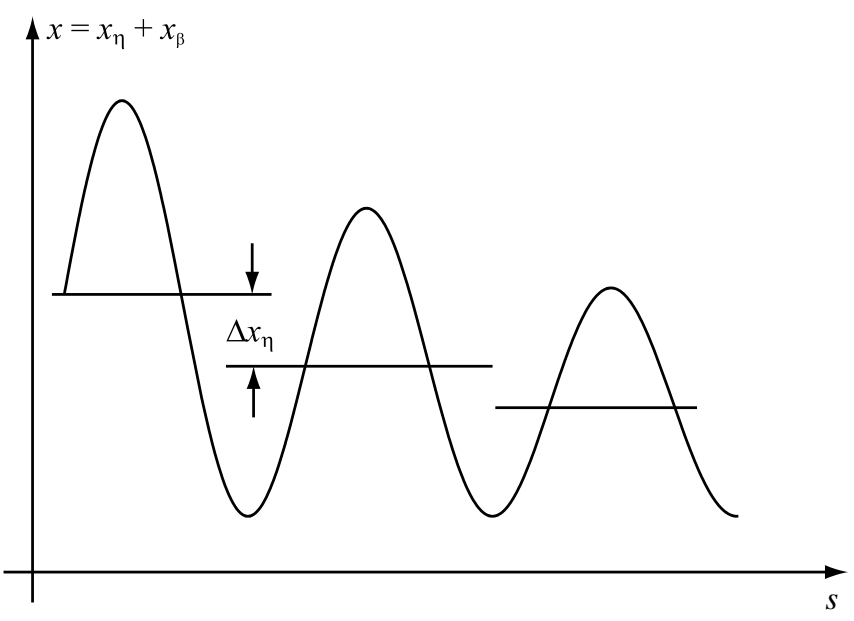

FIG. 2. Motion of a particle in the longitudinal-radial plane. $\Delta x_{\eta}$ is a jump of the closed orbit.

then the deviation of the particle in the kicker undulators becomes $x_{\beta}<0$. In this case the energy loosed by particles is accompanied by a decrease in both energy spread and the amplitudes of betatron oscillations of the beam (see Fig. 2). If the accelerating rf system is switched off, radiation energy losses are neglected, and all particles of the beam are injected in the decelerating phases then, after the movable screen will open images of particles with minimal energy, the optical system must be switched off. Both the spread of closed orbits and amplitudes of betatron oscillations will be decreased and the cooling process can be repeated. The EOC is going both in the longitudinal and transverse degrees of freedom simultaneously. Definite conditions must be fulfilled in the lattice and optical system of the storage ring to inject all particles of the beam in the kicker undulators at decelerating phases during the process of cooling. An optical delay line can be used together with (or without) an isochronous pass-way between undulators for this purpose. Cooling in the bucket can be produced as well.

The screen can be immovable and open only a part of the space for URWs, say $x>x_{T}>0$. In this case the sum of betatron amplitudes and deviations of closed orbits of particles in the cooled beam will be limited by the value $\sim x_{T}$. Another scheme can be used depending on parameters of storage rings, availability of accelerating rf fields in the cooling process, duration of impulses of the laser amplifiers, distances between kicker undulators, delay times for URWs, and so on. EOC in the longitudinal or transverse planes only is possible $[4,5]$.

\section{TO THE FOUNDATIONS OF ENHANCED OPTICAL COOLING}

The total amount of energy carried out by undulator radiation (UR) emitted by electrons traversing an undulator, according to classical electrodynamics, is given by

$$
E_{\mathrm{tot}}^{\mathrm{cl}}=\frac{2}{3} r_{e}^{2} \overline{B^{2}} \beta^{2} \gamma^{2} L_{u}
$$

where $r_{e}=e^{2} / m_{e} c^{2}$ is the classical electron radius; $e, m_{e}$ are the electron charge and mass, respectively; $\overline{B^{2}}$ is an averaged square of the magnetic field along the undulator period $\lambda_{u} ; \beta=v / c$ is the relative velocity of the electron; $\gamma=E / m_{e} c^{2}$ is the relativistic factor; $L_{u}=M \lambda_{u}$ is the length of the undulator; and $M$ is the number of undulator periods. For a planar harmonic undulator $\overline{B^{2}}=B_{0}^{2} / 2$, where $B_{0}$ is the peak of the undulator field. For a helical undulator $\overline{B^{2}}=B_{0}^{2}$. The spectral distribution of the first harmonic of UR for $K<1, M \gg 1$ is given by

$$
d E_{1}^{\mathrm{cl}} / d \xi=E_{1}^{\mathrm{cl}} f(\xi) \quad(0 \leq \xi \leq 1),
$$

where $E_{1}^{\mathrm{cl}}=E_{\mathrm{tot}}^{\mathrm{cl}} /\left(1+K^{2}\right)^{2}, f(\xi)=3 \xi\left(1-2 \xi+2 \xi^{2}\right)$, $\xi=\lambda_{1, \min } / \lambda_{1}, \quad \lambda_{1 \text { min }}=\left.\lambda_{1}\right|_{\theta=0}, \quad \int f(\xi) d \xi=1, \quad K=$ $e \sqrt{B^{2}} \lambda_{u} / 2 \pi m_{e} c^{2}$ is the deflection parameter, $\lambda_{1}=\lambda_{u}(1+$ $\left.K^{2}+\vartheta^{2}\right) / 2 \gamma^{2}$ is the wavelength of the first harmonic of the UR and $\vartheta=\gamma \theta ; \theta$ is the axial angle between the vector of electron average velocity in the undulator and the undulator axis [11].

Electrons have an effective resonant interaction in the field of the kicker undulator only with that part of their undulator radiation wavelets (URW) emitted in the pickup undulator if the frequency bands and the angles of the electron average velocities are selected in the ranges

$$
\left(\frac{\Delta \omega}{\omega}\right)_{C}=\frac{1}{k_{f} M}, \quad(\Delta \vartheta)_{C}=\sqrt{\frac{1+K^{2}}{M}}
$$

nearby maximal frequency and to the axes of both pickup and kicker undulators. The coefficient $k_{f}$ is higher or equal 2. Below we will take $k_{f}=2$. Optical filters which are tuned up to the maximal frequency of the first harmonic of the UR can be used for this selection. In this case, special screens can be used to select the URWs emitted at angles $\vartheta_{\text {URW }}<(\Delta \vartheta)_{C}$ to the pickup undulator axis both in horizontal and vertical directions before they enter optical amplifier (to do away with the unwanted part of URWs loading OPA). The angle between the average electron velocity vector in the undulator and the undulator axis will be small:

$$
(\Delta \vartheta)_{e}<(\Delta \vartheta)_{C}
$$

Below we suggest that the optical system of EOC selects a portion of URWs, emitted in this range of angles and frequencies, by filters, diaphragms, and/or screens. This condition limits the precision $\delta \psi_{x, z}^{\text {bet }}$ of the phase advance $\psi_{x, z}^{\text {bet }}$ determined by the equation

$$
(\delta \theta)_{x, z}^{\text {bet }}<(\Delta \theta)_{C}
$$

where $(\delta \theta)_{x, z}^{\text {bet }}=\left(2 \pi A_{x, z, \text { bet }} / \lambda_{x, z \text {,bet }}\right) \sin \left(\delta \psi_{x, z}^{\text {bet }}\right)$ is the change of the angle between the electron average velocity and the axis of the kicker undulator owing to an error in the 
arrangement of undulators, $A_{x, z \text {, bet }}$ is the amplitude of the betatron oscillations of the electron in the storage ring, in the smooth approximation $\delta \psi_{x, z}^{\text {bet }}=2 \pi \Delta s / \lambda_{x, z \text {, bet }}, \Delta s$ is the displacement of the kicker undulator from optimal position, $\lambda_{x, z \text {,bet }}$ is the length of the period of betatron oscillations.

The number of the photons in the URW emitted by an electron in the suitable for cooling frequency and angular ranges (3) is defined by the following formula (see Appendix A)

$$
N_{\mathrm{ph}}^{\mathrm{cl}}=\frac{\Delta E_{1}^{\mathrm{cl}}}{\hbar \omega_{1 \max }}=\pi \alpha \frac{K^{2}}{1+K^{2}},
$$

where $\quad \Delta E_{1}^{\mathrm{cl}}=\left(d E_{1}^{\mathrm{cl}} / d \omega\right) \Delta \omega_{C}=3 E_{\mathrm{tot}} / 2 M\left(1+K^{2}\right)^{2}$, $\omega_{1 \max }=2 \pi c / \lambda_{1 \text { min }}, \alpha=e^{2} / \hbar c \cong 1 / 137$ [11]. Filtered URWs must be amplified and directed along the axis of the kicker undulator.

If the density of energy in the URWs is approximated by Gaussian distribution with a waist size $\sigma_{w}>\sigma_{x, z}^{e}, Z_{R}>$ $L_{u} / 2$, the rms electric field strength $E_{w}^{\mathrm{cl}}$ of the wavelet of the length $2 M \lambda_{1 \mathrm{~min}}$ in the kicker undulator is defined by the expression

$$
E_{w}^{\mathrm{cl}}=\sqrt{\frac{\Delta E_{1}^{\mathrm{cl}}}{M \sigma_{w}^{2} \lambda_{1 \min }}}=\frac{\sqrt{2} r_{e} \gamma^{2} \sqrt{\overline{B^{2}}}}{\left(1+K^{2}\right)^{3 / 2} \sqrt{M} \sigma_{w}},
$$

where $\sigma_{x, z}^{e}$ is the electron beam dimensions, $Z_{R}=$ $4 \pi \sigma_{w, c}^{2} / \lambda_{1 \text { min }}$ is the Rayleigh length. If $\sigma_{x, z}^{e}<\sigma_{w, c}, \sigma_{W}=$ $\sigma_{w, c}$, the rms electric field strength $E_{w}^{c l}$ of the wavelet becomes

$$
E_{w}^{\mathrm{cl}}=\frac{4 \sqrt{2 \pi} r_{e} \gamma^{3} \sqrt{\overline{B^{2}}}}{L_{u}\left(1+K^{2}\right)^{2}}
$$

where $\sigma_{w, c}=\sqrt{L_{u} \lambda_{1 \min } / 8} \bar{\pi}$ is the waist size corresponding to the Rayleigh length $Z_{R}=L_{u} / 2$.

Note that electric field values (7) and (8) do not take into account the quantum nature of emission of URWs in a pickup undulator. They are valid for $N_{\mathrm{ph}}^{\mathrm{cl}} \gg 1$. Such a case can be realized only for heavy ions at $K Z>1$, where $Z$ is the ion atomic number. For ions in the expression (6), the coefficient $Z^{2}$ must be introduced. If, according to classical electrodynamics, $N_{\mathrm{ph}}^{\mathrm{cl}}<1$, it means that in the reality, according to quantum theory, one photon is emitted with the energy $\hbar \omega_{1, \max }$ and with the probability $p_{\mathrm{em}}=N_{\mathrm{ph}}^{\mathrm{cl}}<$ 1. In this case the electric field strength is determined by the replacement of the energy $\Delta E_{1}^{\mathrm{cl}}$ on $\Delta E_{1}^{q}=$ $\Delta E_{1}^{\mathrm{cl}} / N_{\mathrm{ph}}^{\mathrm{cl}}=\hbar \omega_{1, \max }$ in (7) and the frequency of the emission of URWs $f_{\mathrm{URW}}=f \cdot p_{\mathrm{em}}=f \cdot N_{\mathrm{ph}}^{\mathrm{cl}}<f$, where $f$ is the revolution frequency of the electron in the storage ring.

The maximum rate of energy losses for the electron in the fields of the kicker undulators and amplified URW is

$$
\begin{aligned}
P_{\text {loss }}^{\max } & =-\left.e E_{w}^{\mathrm{cl}} L_{u} \beta_{\perp m} f \Phi\left(N_{\mathrm{ph}}^{\mathrm{cl}}\right) N_{\mathrm{kick}} \sqrt{\alpha_{\mathrm{ampl}}}\right|_{\sigma_{w}=\sigma_{w, c}} \\
& =\frac{8 \pi \sqrt{\pi} e^{2} f \Phi\left(N_{\mathrm{ph}}^{\mathrm{cl}}\right) N_{\mathrm{kick}} K^{2} \sqrt{\alpha_{\mathrm{ampl}}}}{\left(1+K^{2}\right) \lambda_{1, \min }}
\end{aligned}
$$

where $\beta_{\perp m}=K / \gamma, N_{\text {kick }}$ is the number of kicker undulators, $\alpha_{\text {ampl }}$ is the gain in the optical amplifier. The function $\left.\Phi\left(N_{\mathrm{ph}}^{\mathrm{cl}}\right)\right|_{N_{\mathrm{ph}}^{\mathrm{cl}} \ll 1}=\sqrt{p_{\mathrm{em}}}=\sqrt{N_{\mathrm{ph}}^{\mathrm{cl}}}$ takes into account the quantum nature of the URWs emission at $N_{\mathrm{ph}}^{\mathrm{cl}} \ll 1$ : the frequency of emission of URWs $\sim f \cdot N_{\mathrm{ph}}^{\mathrm{cl}}$ and the electric field strength $\left.\sim E_{w}^{q}\right|_{N_{\mathrm{ph}}<1}=E_{w}^{\mathrm{cl}} / \sqrt{N_{\mathrm{ph}}^{\mathrm{cl}}}$. It follows that quantum nature of the photon emission in undulators leads to the decrease of the maximum average rate of energy losses for electrons in the fields of the kicker undulator and amplified URW by the factor $\left.\Phi\left(N_{\mathrm{ph}}^{\mathrm{cl}}\right)\right|_{N_{\mathrm{ph}}^{\mathrm{cl}} \ll 1}=$ $\sqrt{\left.N_{\mathrm{ph}}^{\mathrm{cl}}\right|_{K=1, Z=1}} \simeq 0.107$. For ions at $K Z>1$ the function $\left.\Phi\left(N_{\mathrm{ph}}^{\mathrm{cl}}\right)\right|_{N_{\mathrm{ph}}^{\mathrm{cl}}>1}=1$.

The damping times for the longitudinal and transverse degrees of freedom are

$$
\tau_{s, \mathrm{EOC}}=\frac{6 \sigma_{E, 0}}{\left|P_{\mathrm{loss}}^{\max }\right|}, \tau_{x, \mathrm{EOC}}=\tau_{s, \mathrm{EOC}} \frac{\sigma_{x, 0}}{\sigma_{x_{\eta}, 0}}=\frac{6 \beta^{2} E_{s} \sigma_{x, 0}}{\left|P_{\mathrm{loss}}^{\max }\right| \eta_{x, \mathrm{kick}}},
$$

where $\sigma_{E, 0}$ is the initial energy spread of the electron beam, $\sigma_{x, 0}$ is the initial radial beam dimension determined by betatron oscillations, $\eta_{x \text {,kick }} \neq 0$ is the dispersion function in the kicker undulator, $\sigma_{x_{\eta}, 0}=\eta_{x, \text { kick }} \beta^{-2}\left(\sigma_{E, 0} / E\right)$. Note that the damping time for the longitudinal direction does not depend on $\eta_{x \text {,ick }}$ and one for the transverse

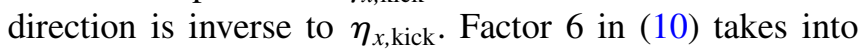
account that the energy spread for cooling is $2 \sigma_{E, 0}$, electrons meet less often with their URWs (screening effect), and that the jumps of the electron energy and closed orbit in general case lead to lesser jumps of the amplitude of synchrotron and betatron oscillations [6]. It does not take into account the decrease in efficiency of the interaction of the electron with its URW if the angular and the energy spreads of the beam are higher the limiting values [see (4) and (14)].

The equilibrium beam dimensions $\sigma_{x_{\eta}}^{\mathrm{EOC}}, \sigma_{x}^{\mathrm{EOC}}$, corresponding spreads in the positions of the closed orbits and in betatron amplitudes at $N_{\mathrm{ph}}^{\mathrm{cl}}<1$ and at the number of photons produced by $N_{s}-1$ extraneous electrons and noise photons in the sample $N_{\mathrm{ph}, \Sigma}^{\mathrm{cl}} \simeq N_{\mathrm{ph}}^{\mathrm{cl}}\left(N_{s}-1\right)+N_{n}>1$ are

$$
\begin{aligned}
\sigma_{x, \mathrm{eq}}^{\mathrm{EOC}} & =\sigma_{x_{\eta}, \mathrm{eq}}^{\mathrm{EOC}}=\left(\sqrt{\overline{A_{x}^{2}}}\right)_{\mathrm{eq}}^{\mathrm{EOC}} / \sqrt{2}=\left(\sqrt{\overline{x_{\eta}^{2}}}\right)_{\mathrm{eq}}^{\mathrm{EOC}} /\left.\sqrt{2}\right|_{N_{\mathrm{ph}, \mathrm{c}}^{\mathrm{cl}}>1} \\
& =\frac{1}{2 \sqrt{2}}\left(N_{e, s}-1+N_{n} / N_{\mathrm{ph}}^{\mathrm{cl}}\right)\left|\delta x_{\eta}^{1}\right|
\end{aligned}
$$

where $\delta x_{\eta}^{1}=\eta_{x, \text { kick }} \beta^{-2}\left(\Delta E_{\text {loss }}^{\max } / E\right)$ is the jump of the electron closed orbit determined by the energy jump 
$\Delta E_{\text {loss }}^{\max }=P_{\text {loss }}^{\max } / f N_{\mathrm{ph}}^{\mathrm{cl}}$ of the electron in the fields of the kicker undulator and its amplified URW, $N_{e, s}$ is the number of electrons in the URW sample, $N_{n}$ is the number of noise photons in the URW sample at the amplifier front end [6,7]. Note that the energy jump $\Delta E_{\text {loss }}^{\max }$ corresponds to emission by signal electron in the pickup undulator of one-photon in the URW. In our case $N_{e, s}=2 M \lambda_{1, \min } N_{e} / \sigma_{s, 0}, N_{e}$ stands for the number of electrons in the bunch, $\sigma_{s, 0}$ is the initial length of the electron bunch.

The equilibrium relative energy spread of the electron beam $\sigma_{E \text {,eq }}^{\mathrm{EOC}}=\beta^{2} \sigma_{x_{\eta} \text {,eq }}^{\mathrm{EOC}} E / \eta_{x, \text { kick }}$ or

$$
\sigma_{E, \text { eq }}^{\mathrm{EOC}}=\frac{1}{2 \sqrt{2}}\left(N_{e, s}-1+N_{n} / N_{\mathrm{ph}}^{\mathrm{cl}}\right) \Delta E_{\mathrm{loss}}^{\max } .
$$

The power of the optical amplifier is determined by the power transferred to the electron beam and by a noise power:

$$
P_{\text {ampl }} \leq \varepsilon_{\text {sample }}^{\mathrm{cl}} f N_{e} / 2+P_{n}
$$

where $\varepsilon_{\text {sample }}^{\mathrm{cl}}=\Delta E_{1}^{\mathrm{cl}} \alpha_{\mathrm{ampl}}=\hbar \omega_{1, \max } N_{\mathrm{ph}}^{\mathrm{cl}} \alpha_{\mathrm{ampl}}$ is the average energy in the amplified URW. This power corresponds to the case in which half of the total number of electrons are involved in the cooling process simultaneously (screening is introduced) and the amplification time interval of the amplifier is higher than the time duration of the electron bunch: $\Delta t_{\text {ampl }}>\Delta t_{b}$.

The initial phases $\varphi_{\text {in }}$ of electrons in their URWs radiated in the pickup undulator and transferred to the entrance of the kicker undulator(s), in the general case, depend on their energies and amplitudes of betatron oscillations. Name the electron with zero amplitude of betatron oscillations and a dedicated energy $E_{d}$ a signal one if it enters the kicker undulator together with its URW and receive maximal energy. Other electrons, depending on the initial amplitude and initial energy, will enter the pickup undulator nonsimultaneously at accelerating or decelerating phases and receive lesser energy. All electrons will get acceleration, if the orbit lengths between undulators for the signal and other electrons differ less than $\lambda_{1, \min } / 2$. It follows that the energy spread, the amplitudes of betatron oscillations, and the transverse horizontal emittance of such a beam in the smooth approximation must not exceed the values

$$
\begin{aligned}
\frac{\sigma_{E}}{E} & <\left(\frac{\sigma_{E}}{E}\right)_{\lim }=\frac{\lambda_{1 \mathrm{~min}}}{2 L_{p, k}} \frac{\beta^{2}}{\eta_{c, l}}, \\
A_{x} & <A_{x, \mathrm{lim}}=\sqrt{\lambda_{1 \mathrm{~min}} \lambda_{x, \mathrm{bet}}} / \pi, \\
\varepsilon_{x} & <2 \lambda_{1 \mathrm{~min}} / \pi,
\end{aligned}
$$

where $L_{p, k}$ is the distance between pickup and kicker undulators along the synchronous orbit, $\eta_{c, l}=$ $-d \ln T_{p, k} / d \ln p$ is the local slippage factor between the undulators, $p$ is the momentum of an electron, $T_{p, k}=$
$L_{p, k} / c \boldsymbol{\beta}$ is the pass by time between pickup and kicker undulators. In accordance with the betatron phase advance $\psi_{x}^{\text {bet }}=2 \pi\left(k_{p, k}+1 / 2\right)$, the value $L_{p, k}=\lambda_{x, \text { bet }}\left(k_{p, k}+\right.$ $1 / 2)$, where $\lambda_{x, \text { bet }}=C / v_{x}$ is the wavelength of betatron oscillations, $C$ is the circumference of the ring, $v_{x}$ is the betatron tune.

The first equation in (14) can be overcome if the isochronous bend or bypass between undulators will be used. In some cases a controllable variable in the time optical delay line can be used to change in situ the length of the light pass-way between the undulators during the cooling cycle to keep the decelerating phases of electrons in the kicker undulator in the process of cooling [6,7].

Below we investigate this case in more detail: The difference $\Delta t$ in the propagation time of the URW and the traveling time $T_{p, k}$ of the electron between pickup and kicker undulators depends on initial conditions of the electron's trajectory which can be expressed as

$$
\begin{aligned}
\left.c \Delta t\right|_{\gamma \gg 1}= & c_{t}-\int_{s 0}^{s} \frac{x}{R} d \tau \\
= & c_{t}-x_{0} \int_{s 0}^{s} \frac{C}{R} d \tau-x_{0}^{\prime} \int_{s 0}^{s} \frac{S}{R} d \tau \\
& -\frac{\Delta E}{\beta^{2} E} \int_{s 0}^{s} \frac{D}{R} d \tau,
\end{aligned}
$$

where $x=x_{\boldsymbol{\beta}}+x_{\boldsymbol{\eta}}, x_{0}=x_{0 \boldsymbol{\beta}}+x_{0 \boldsymbol{\eta}}, x_{\boldsymbol{\beta}}$ is the deviation of the electron from its closed orbit, $x_{\boldsymbol{\eta}}$ is the deviation of the closed orbit itself from the synchronous one, $x_{0 \boldsymbol{\beta}}$ and $x_{0 \eta}$ stand for appropriate deviations at location $s=s_{0}, \Delta E=$ $E-E_{d}$ is the deviation of the electron energy from the dedicated energy $E_{d}$. Two eigenvectors are called sinelike $S(z)$ and cosinelike $C(z)$ trajectories and $R$ stands for the local bending radius, $c_{t}$ is a constant which is determined by the optical delay line. Basically vectors $S(z), C(z)$ describe the trajectories with initial conditions like $x_{0}^{\prime}\left(s_{0}\right)=0 ; \quad x\left(s, s_{0}\right)=x_{0} C\left(s, s_{0}\right) \quad$ and $\quad x_{0}\left(s_{0}\right)=0$; $x\left(s, s_{0}\right)=x_{0}^{\prime} S\left(s, s_{0}\right)$, where $s_{0}$ corresponds to the longitudinal position of the pickup. So the transverse position of the particle has the form [12]

$$
x(s)=x_{0} C\left(s, s_{0}\right)+x_{0}^{\prime} S\left(s, s_{0}\right)+D\left(s, s_{0}\right)\left(\boldsymbol{\Delta} E / \boldsymbol{\beta}^{2} E\right),
$$

where dispersion $D$ is defined as

$$
D\left(s, s_{0}\right)=-S\left(s, s_{0}\right) \int_{s 0}^{s} \frac{C(\tau)}{R} d \tau-C\left(s, s_{0}\right) \int_{s 0}^{s} \frac{S(\tau)}{R} d \tau .
$$

Dispersion $D\left(s, s_{0}\right)$ describes the transverse position of the test particle having relative momentum deviation from equilibrium as big as $\Delta p / p$, while its initial values of transverse coordinates at $s=s_{0}$ are zero. So full expression for transverse position of particle comes to form

$$
\begin{aligned}
x(s)= & x_{0 \beta} C\left(s, s_{0}\right)+x_{0 \beta}^{\prime} S\left(s, s_{0}\right) \\
& +\left[\eta_{x, 0} C\left(s, s_{0}\right)+\eta_{x, 0}^{\prime} S\left(s, s_{0}\right)+D\left(s, s_{0}\right)\right] \frac{\Delta E}{\beta^{2} E},
\end{aligned}
$$


where $\eta_{x}$ describes the periodic solution for dispersion in the damping ring (slippage factor) and $x_{0 \boldsymbol{\beta}}, x_{0 \boldsymbol{\beta}}^{\prime}$ mark pure the betatron part in the transverse coordinate; $\eta_{0, x}, \eta_{0, x}^{\prime}$ stand for its value at location of the pickup undulator. So the time difference becomes

$$
\begin{aligned}
c \Delta t= & {\left[c_{t}-R_{51}\left(s, s_{0}\right) x_{0}-R_{52}\left(s, s_{0}\right) x_{0}^{\prime}\right.} \\
& \left.-R_{56}\left(s, s_{0}\right) \frac{\Delta E}{\beta^{2} E}\right]\left.\right|_{R_{51}=R_{52}=0} \\
= & c_{t}+c T_{p, k} \eta_{c, l} \frac{\Delta E}{\beta^{2} E} .
\end{aligned}
$$

Elements of transport matrix $R_{i, j}$ have their usual meaning clear from previous equations. In the general case $\eta_{c, l} \neq 0$, $R_{51}=R_{52} \neq 0, A_{x}>0, A_{z}>0, \sigma_{E, 0} \neq 0$, the initial phase of an electron in the field of amplified URW propagating through the kicker undulator, according to (15), $\varphi_{\text {in }}=$ $\omega_{1, \text { max }} \Delta t=\omega_{1, \max }\left(\Delta t_{\text {bet }}+\Delta t_{\text {disp }}\right)=\varphi_{\text {in,bet }}+\varphi_{\text {in,disp }} \neq 0$ and the rate of the energy loss

$$
P_{\text {loss }}=P_{\text {loss }}^{\max } f\left(\Delta E, A_{x}, A_{z}\right) \cos \left(\varphi_{\text {in }}\right),
$$

where $f\left(\Delta E, A_{x}, A_{z}\right)=1-\left|\varphi_{\text {in }}\left(\Delta E, A_{x}, A_{z}\right)\right| / 2 \pi M$, if $\left|\varphi_{\text {in }}\right| \leq 2 \pi M$ and $f\left(\Delta E, A_{x}, A_{z}\right)=0$ if $\left|\varphi_{\text {in }}\right|>2 \pi M$, $\varphi_{\text {in, bet }}, \varphi_{\text {in,disp }}$ are phase shifts responsible for betatron oscillations and the energy deviation $\Delta E=E-E_{d}$. The function $f\left(\Delta E, A_{x}, A_{z}\right)$ takes into account that the electron with small amplitude of betatron oscillations $A_{x} \ll A_{x, \text { lim }}$ [see (14)] or at $R_{51}=R_{52}=0$ and the energy $E_{d}$ enter the kicker undulator simultaneously with its URW at the phase $\varphi_{\text {in }}=0, c_{t}=0$ and pass all undulator lengths at the maximum rate of the energy gain.

The power loss $P_{\text {loss }}$ is the oscillatory function of the energy $\left|E-E_{d}\right|$ with the amplitude linearly decreasing from the maximum value $\left|P_{\text {loss }}^{\max }\right|$ at the energy $E=E_{d}$ to a zero one at the energy $\left|E-E_{d}\right| \geq M \delta E_{\text {gap }}$ and $A_{x}=$ $A_{z}=0$ [see below (18)]. Electrons having the energies $E \neq E_{d}$ and their URWs enter the kicker undulator nonsimultaneously with different phases and travel together a shorter distance in the undulator. According to (16), they are accelerated or decelerated and gathered at phases $\varphi_{m}=$ $\pi / 2+2 \pi m, m=0, \pm 1, \ldots \pm(M-1)$, and at energies

$$
\begin{aligned}
E_{m} & =E_{d}+\frac{(2 m+1) \pi \beta^{2}}{\omega_{1, \max } T_{p, k} \eta_{c, l}} E_{d} \\
& =E_{d}\left[1+\frac{(2 m+1) \lambda_{1, \min } \beta^{2}}{2 L_{p, k} \eta_{c, l}}\right],
\end{aligned}
$$

if the rf accelerating system is switched off and betatron oscillations are neglected (see Fig. 3). Here $L_{p, k}=c \beta T_{p, k}$.

The energy gaps between equilibrium energy positions have magnitudes given by

$$
\delta E_{\text {gap }}=E_{m+1}-E_{m}=\frac{\lambda_{1, \min }}{L_{p, k} \eta_{c, l}} \beta^{2} E_{d} .
$$

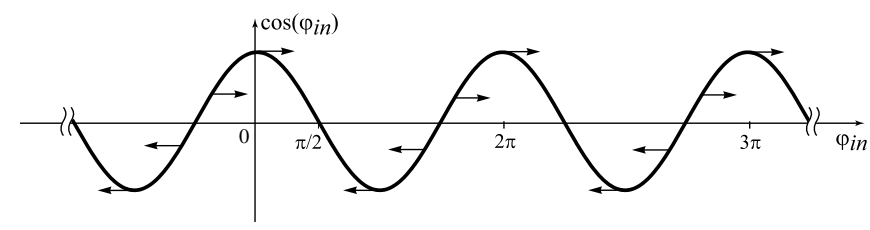

FIG. 3. In the EOC scheme electrons are grouping near the phases $\varphi_{\text {in }}=\pi / 2+2 \pi m$ (energies $\left.E_{m}\right)$.

If the rf accelerating system is switched off, the electron energy falls into the energy range $\left|E-E_{d}\right|<M \delta E_{\text {gap }}$, the number of particles in the sample is small, the multiple processes of excitation of synchrotron oscillations by extraneous URWs and noise photons does not move out electrons from the gaps then the electron energy is drifting to the nearest value $E_{m}$. The last condition is valid if the equilibrium energy spread of the beam

$$
\sigma_{E \text {,eq }}^{\mathrm{EOC}} \ll \delta E_{\text {gap }}
$$

The variation of the particle's energy looks like it produces aperiodic motion in one of $2 M$ potential wells located one by one. The depth of the wells is decreased with the number $|m|$.

If the delay time in the optical line is changed, the energies $E_{m}$ and the energies of particles in the well are changed as well. In this case particles stay in their wells if their maximal power loss

$$
\left|P_{\text {loss }}^{\max }\right|>\left|d E_{m} / d t+P_{\text {loss }}^{\text {ext }}\right|,
$$

where $\left|P_{\text {loss }}^{\text {ext }}\right|$ stands for the external power losses determined by synchrotron radiation.

We will take below the synchronous energy of the electron $E_{s}=E_{d}+E_{m=0}=E_{d}+\delta E_{\text {gap }} / 2$.

\section{VARIANTS OF OPTICAL COOLING}

Different variants of optical cooling of particle beams can be suggested depending on the local slippage factor, coefficients $R_{51}, R_{52}$, and $R_{56}$ in (14), dispersion and beta functions of the storage ring in the pickup and kicker undulators, duration of the impulse of the laser amplifier. Below we present some of them.

1. The local slippage factor $\boldsymbol{\eta}_{c, l}=0$, amplitudes of betatron oscillations satisfy the equations (14) $\left(\left|\varphi_{\text {in }}\right|<\right.$ $\pi / 2$ ), dispersion function in the pickup undulator $\eta_{x \text {,pickup }} \neq 0$. In this case the difference in the propagation time of the URW and the traveling time $T_{p, k}$ of the electron between pickup and kicker undulators is $\Delta t \simeq$ const. Hence, the initial phase can be installed $\varphi_{\text {in }} \simeq \pi$ for all electrons. It corresponds to electrons arriving in the kicker undulator in decelerating phases of their URWs under a maximum rate of energy loss. Electrons will be gathered near the synchronous electron if a moving screen opens the way only to URWs emitted by electrons with the energy higher than the synchronous one. This is the case of an 
EOC both in the longitudinal and the transverse plane based on an isochronous bend and screening technique.

If the dispersion function in the pickup undulator $\eta_{x}=$ 0 or if the synchrotron oscillations of electrons are small (no selection in the longitudinal plane), a moving screen opens the way only to URWs emitted by electrons with deviations $x=x_{\eta}+x_{b} \geq 0$, then the cooling takes place only in the transverse direction. In this case the amplitudes of betatron oscillations of electrons are decreased at every interaction with their URWs but the amplitude of the synchrotron oscillations is not changed in average in the first approximation.

If the initial energy spread of the electron beam is small $\left(\left|\varphi_{\text {in,disp }}\right| \ll 1\right)$, amplitudes of betatron oscillations of electrons at given energy exceed the limiting one (14), $R_{51}=$ $R_{52} \neq 0$, and that is why their initial phases are distributed in a wide region $\left|\varphi_{\text {in }}\right|=\left|\varphi_{\text {in, bet }}\right|>2 \pi$, the EOC will be accompanied by grouping of electrons at phases $\varphi_{m}=$ $\pi / 2+2 \pi m$ and amplitudes

$$
A_{n}=\sqrt{2 n \lambda_{b} \lambda_{1, \min }} / \pi
$$

corresponding to the excess of the length of the real orbit of the electron over the length of its closed orbit between pickup and kicker undulators $\Delta s=c \Delta t=n \lambda_{1, \min }$, where $n=0,1,2 \ldots$ To decrease the amplitudes of betatron oscillations $\left.A_{n}\right|_{n>1}$, the next cooling cycles must start with the initial decelerating phases $\varphi_{\text {in }} \simeq \pi$ for the electrons with these amplitudes and using variable time delay.

The jumps of amplitudes of synchrotron oscillations are maximal if the deviation of the electron from its synchronous position in the radial direction is maximal. That is why the pulsed laser amplifier must be switched on when URWs are emitted by electrons located near to the synchronous one.

2. The scheme of OSC can be used at $\boldsymbol{\eta}_{c, l}=0$ [8]. In this scheme the pickup undulator is a quadrupole one and the kicker undulator is an ordinary one. They have the same period. The magnetic field in the quadrupole undulator is increased with the radial coordinate $x$ by the low $B(x) \cong$ $G x$ and changes the sign at $x=0$, where $G$ stands for the gradient. The phase of the emitted URWs changes its value on $\pi$ at $x=0$ as well. That is why electrons with the deviation $x>0$ are decelerated and electrons with the deviation $x<0$ are accelerated in the ordinary pickup undulator. It means that they are gathered around a synchronous orbit in the ring where they do not emit URWs.

The deflection parameter in the pickup undulator is increased with the radial coordinate $(K \propto|B(x)|)$ and so the emitted wavelength also. The deflection parameter in the kicker undulator is a constant. It means that the resonance interaction of the electron and URW emitted by this electron is possible in the kicker undulator only at $K \ll 1$ or, in case of $K \simeq 1$, at the narrow region of positions $x_{\eta}$, where the deflection parameters of undulators are near the same. The last case opens a possibility for initial selection of amplitudes in the pickup undulator suitable for cooling and to preserve the electron beam from heating.

The continuous resonance interaction and cooling is possible if the magnetic field of kicker undulator is decreased in time for cooling process.

The scheme with two quadrupole undulators (pickup and kicker ones) is described in [13]. In this case the second quadrupole undulator decreases the amplitudes of synchrotron oscillations for positive deviations $x_{\eta \text {, pickup }}>0$ and increases them for negative ones. To cool the electron beam in this case the additional selection of URWs can be used by the screen (cut off URWs emitted by electrons at negative deviations $x$, EOC scheme).

Another scheme based on the truncated pickup undulator with the magnetic field of the form $\left.B(x)\right|_{x>0} \simeq G x$, $\left.B(x)\right|_{x<0} \simeq 0$ and quadrupole kicker undulator can be used. The pickup undulator can be linearly polarized with one upper array of magnetic poles. Such a kicker undulator was used in the undulator radiation experiments [14].

Betatron oscillations in this scheme must introduce the phase shift $<\pi / 2$. This condition can be arranged by proper zeroing cos- and sin-like trajectory integrals [13].

3. If $\eta_{c, l} \neq 0$, betatron oscillations of electrons introduce phase shift $<\pi / 2$ and the energy gaps have the magnitude $\delta E_{\text {gap }}=(3 \div 5) \sigma_{E, 0}$, where $\sigma_{E, 0}$ is the initial energy spread of the electron beam, then the transit-time method of OSC based on two identical undulators can be used [9]. In this case the initial phase for a synchronous electron in the URW can be installed $\varphi_{\mathrm{in}, s} \simeq \pi / 2$. It corresponds to the electron crossing the kicker undulator under a zero rate of the energy loss. The higher the deviation of the initial phases of other electrons from the synchronous one, the higher the rate of the energy loss $\left(\sim \sin \varphi_{\text {in }}\right)$. In this case the main part of the electrons including tail electrons will be gathered at the energy $E_{s}$. The time depended local slippage factor can be used to decrease the energy gap during the cooling process in order to increase the rate of cooling at small amplitudes of phase and betatron oscillations.

4. If $\eta_{c, l} \neq 0$, the energy gaps between equilibrium energy positions have the magnitudes $\delta E_{\text {gap }} \cong$ $(3 \div 5) \sigma_{E .0} / M, \sigma_{E \text {,eq }}^{\mathrm{EOC}} \ll \delta E_{\text {gap }}$ [see (19)], rf accelerating system of the storage ring is switched off, the screen overlap URWs emitted by electrons at negative deviation from the closed orbit corresponding to electrons having minimum energy $E_{\min }$, then electrons are gathered at phases of URWs $\varphi_{m}=\pi / 2+2 \pi m$ and, in the general case $R_{51} \neq 0, R_{52} \neq 0, A_{x}>0, A_{z}>0$, at energies and amplitudes, which, strictly speaking, differ from $E_{m}$ and $A_{n}$ [see (17) and (21)] but near to them. After that, if the optical system changes the delay time of the URWs to move the energies of electrons $E>E_{\min }$ to the energy $E_{\min }$, then electrons lose their energy and amplitudes of 
betatron oscillations until their energy takes minimum one. Cooling takes place according to the scheme of the EOC. The cooling process must be repeated with the initial decelerated phases $\varphi_{\text {in }} \simeq \pi$ using variable time delay.

5. If $\eta_{c, l} \neq 0$, the energy gaps between equilibrium energy positions have the magnitudes $\delta E_{\text {gap }} \ll \sigma_{E, 0} / M$, $\sigma_{E \text {,eq }}^{\mathrm{EOC}} \ll \delta E_{\text {gap }}$, the rf accelerating system is switched off, the screen overlap URWs emitted by electrons at negative deviation from closed orbit corresponding to electrons of the beam having minimum energy $E_{\min }$ and optical system changes the delay time of the URWs to move the energies of electrons $E>E_{\min }$ to the energy $E_{\min }$, then electrons with smaller energy are captured increasingly in the regions of the energy layers $E_{m}$ and lose their energy and amplitudes of betatron oscillations until their energy takes minimum one. Cooling takes place according to the scheme of the EOC. This process can be repeated.

6. If $\eta_{c, l} \neq 0$, the energy gaps between equilibrium energy positions have the magnitudes $\delta E_{\text {gap }} \ll \sigma_{E, 0} / M$, $\sigma_{E \text {,eq }}^{\mathrm{EOC}} \ll \delta E_{\text {gap }}$, rf accelerating system of the storage ring is switched on, the screen overlap URWs emitted by electrons at negative deviation from synchronous closed orbit, the average energy loss per turn $\Delta E_{\text {loss }}^{\text {turn }}=\left|P_{\text {loss }}^{\max }\right| / f$ is higher than the energy gain $\left|e V_{0}\left(\sin \phi-\sin \phi_{s}\right)\right|$ of the electron in the rf accelerating system of the storage ring or if

$$
\left|\sin \phi-\sin \phi_{s}\right| \leq \delta E_{\text {loss }}^{\text {turn }} / e V_{0},
$$

then the phases of electrons in their URWs are drifting to the nearest phases $\varphi_{m}=\pi / 2+2 \pi m$ [the corresponding energies and amplitudes in this case differ from $E_{m}$ and $A_{n}$ determined by (17) and (21)]. Here $V_{0}$ is the amplitude of the rf accelerating voltage, $\phi_{s}$ is the synchronous phase determined by the equation $\sin \phi_{s}=\Delta E_{\mathrm{SR}, s} / e V_{0}=$ $V_{s} / V_{0}, \Delta E_{\mathrm{SR}, s}=P_{\mathrm{SR}, s} / f=e V_{s}$ is the synchrotron radiation energy loss of synchronous electron per turn, $P_{\mathrm{SR}, s}=$ $\frac{2}{3} c r_{e}^{2} \bar{B}^{2} \gamma^{2} \simeq 2.77 \times 10^{3} \gamma^{4} / R_{s} \overline{R_{s}} \mathrm{eV} / \mathrm{sec}$ is the average power of the synchrotron radiation emitted by the electron in the storage ring. The value $P_{\mathrm{SR}, s} / f=5.8 \times$ $10^{-7} \gamma^{4} / R_{s} \mathrm{eV} /$ turn, $R_{s}$ is the bending radius of the synchronous particle, $\overline{R_{s}}$ is the average bending radius of the ring.

To keep the condition (22) satisfied at $e V_{0}>\delta E_{\text {loss }}^{\text {turn }}$, the range of rf phases of particles $2\left|\phi-\phi_{s}\right|$ interacting with their URWs must be limited by the value $2\left|\phi_{c, 1}-\phi_{s}\right|$ determined by equality in (22). This can be done by using OPA with short amplification time interval $\Delta t_{\text {ampl,1 }}$ corresponding to the range of phases $\omega_{\mathrm{rf}} \Delta t_{\mathrm{ampl}}<2\left|\phi_{c, 1}-\phi_{s}\right|$ and by overlapping the center of this time interval with synchronous particle, where $\omega_{\mathrm{rf}}=2 \pi f_{\mathrm{rf}}, f_{\mathrm{rf}}$ is the frequency of the rf accelerating system of the ring. The last condition is equivalent to

$$
l_{\mathrm{ampl}}^{\mathrm{laser}}<l_{\mathrm{ampl}, 1}=2 c\left|\phi_{c, 1}-\phi_{s}\right| / \omega_{\mathrm{rf}},
$$

where $l_{\text {ampl, } 1}$ is the length of the amplified laser bunch. In this case, depending on the initial phases $\varphi_{\text {in }}$, the electrons will be accelerated or decelerated until they reach the equilibrium phases $\varphi_{m}=\pi / 2+2 \pi m$ and corresponding energies and amplitudes of betatron oscillations $E_{m}$ and $A_{n}$.

$2 M$ electron ellipses appear around synchronous phase $\phi_{s}$ of the storage ring in the longitudinal plane if conditions (19), (22), and (23) are fulfilled, and if the deviation of the electron energy $\delta E=E-E_{m}$ from its equilibrium one $E_{m}$ at the interaction moment is small. The last condition can be overcame by limiting the amplification time of laser amplifier by the interval $\Delta t_{\mathrm{ampl}, 2}$ and the corresponding length $l_{\mathrm{ampl}, 2}=c \Delta t_{\mathrm{ampl}, 2}$ to

$$
l_{\text {ampl }}^{\text {laser }}<l_{\text {ampl }, 2}^{\text {laser }}=\frac{\sigma_{s, 0}}{2 \sqrt{2}} \sqrt{\frac{\delta E_{\text {gap }}}{\sigma_{E, 0}}},
$$

where $\sigma_{s, 0}$ is the initial length of the electron bunch. Above we suggested that electrons are moving along elliptical trajectories $\Delta E= \pm \sigma_{E, 0} \sqrt{1-s^{2} / \sigma_{s, 0}^{2}}$ and interact with URWs at the top energies $\sim E_{m}$ in the region of the energy deviations $\delta E=\left|E-E_{m}\right|=\delta E_{\text {gap }} / 8$, where $s$ is the deviation of electron from synchronous one.

The equilibrium energies and amplitudes of betatron oscillations $E_{m}$ and $A_{n}$ are moved to synchronous energy and zero amplitude of betatron oscillations if the optical system decrease the delay time of the URWs.

Damping time (10) in variant 6 will be increased $2 \sigma_{s, 0} / l_{\mathrm{ampl}}$ times:

$$
\tau_{s}^{\mathrm{EOC}}=\frac{12 \sigma_{E, 0}}{\left|P_{\mathrm{loss}}^{\max }\right|} \frac{\sigma_{s, 0}}{l_{\mathrm{ampl}}} \quad \tau_{x}^{\mathrm{EOC}}=\frac{12 \beta^{2} E_{s} \sigma_{x, 0}}{\left|P_{\mathrm{loss}}^{\max }\right| \eta_{x, \mathrm{kick}}} \frac{\sigma_{s, 0}}{l_{\mathrm{ampl}}} .
$$

The variant 6 permits one to avoid any changes in the existing lattice of the ring (isochronous bend, bypass). It works easier for existing ion storage rings (see Appendix B).

The screen permits one to select in pickup undulator electrons with positive deviations of both betatron and synchrotron oscillations, and such a way to produce effective cooling both in the transverse and longitudinal direction. Using the number of kicker undulators $N_{\text {kick }}>1$ permits one to cool the beam either in two directions or in one (transverse or longitudinal) direction by selecting distances between kicker undulators and time delays of the optical line $[4,6]$.

\section{OPTICAL SYSTEM FOR THE EOC SCHEME}

UR of an electron gets its well-known properties only after the electron passed the undulator and UR is considered in the far zone. The lens located near the pickup undulator can strongly influence the UR properties if its focus is inside the undulator [15]. 
For effective cooling of an electron beam in a storage ring, parameters of the beam under cooling and the optics of EOC system must fulfill certain requirements.

1. Each electron in a beam should enter the kicker undulator simultaneously with its amplified URW and move in the decelerating phase of this URW. For the test electron of a beam (for example, for the synchronous electron with zero amplitude of betatron oscillations), this requirement is satisfied by equating the propagation time of the URW with the traveling time of the electron between undulators. Conditions (14) are necessary for other electrons of the beam to get decelerating phases of theirs URWs in this case.

2. Each electron in the beam should enter the kicker undulator with its URW near the center of this URW in the transverse direction. This requirement will be satisfied if the transverse sizes of all URWs in the kicker undulator are overlapped.

3. The rms transverse size of one URW at the distance $l$ from the pickup undulator is equal to $\sigma_{w} \simeq(\Delta \theta)_{c} \times$ $\left(l+M \lambda_{u} / 2\right)$, where $(\Delta \theta)_{c}$ is determined by (3). If the optical screen opens the way to radiation from the part of the electron beam, so only small angles to the undulator axis $\Delta \vartheta<(\Delta \vartheta)_{C}$ passed through, then URWs will be overlapped at the distance from the end of the undulator

$$
l_{c}=\frac{d}{(\Delta \theta)_{c}},
$$

where $d$ is the transverse size of the electron beam (see Fig. 4). Electrons under cooling will hit their URWs in the transverse plane if the transverse dimensions of the electron beam in the undulator are less than $2 \sigma_{w, b}=2 d+$ $\left.2 \sigma_{w}\right|_{l=l_{c}}$.

4. Generally, the angular resolution of an electron bunch by an optical system is

$$
\delta \varphi_{\mathrm{res}} \cong 1.22 \frac{\lambda_{1 \mathrm{~min}}}{D},
$$

where $D$ is diameter of the first lens. This formula is valid, if elements of the source emit radiation which is distributed uniformly in a large solid angle. In our case only a fraction of the lens is affected by radiation, as usually $D>2 \sigma_{w, b}$. That is why the effective diameter $D_{\text {eff }}=2 \sigma_{w, b}$ must be used in (27). At the distances $l>l_{c}$ the size $\sigma_{w, b} \simeq(\Delta \theta)_{c} l$, so the space resolution of the optical system is $\delta x_{\text {res }} \cong$ $\delta \varphi_{\text {res }} l$ or

$$
\delta x_{\text {res }} \cong 1.22 \lambda_{1 \mathrm{~min}} /(\Delta \theta)_{c}=0.86 \sqrt{\lambda_{1 \mathrm{~min}} L_{u}},
$$

where $(\Delta \theta)_{c}=(\Delta \vartheta)_{c} / \gamma=(1 / \gamma) \sqrt{\left(1+K^{2}\right) / M}$ is the observation angle.

5. URWs must be focused on the crystal of OPA. For the URW beam, the dedicated optical system with focusing lenses can be used to make the Rayleigh length larger to the length of the crystal (typically $\sim 1 \mathrm{~cm}$ ) for small diameters

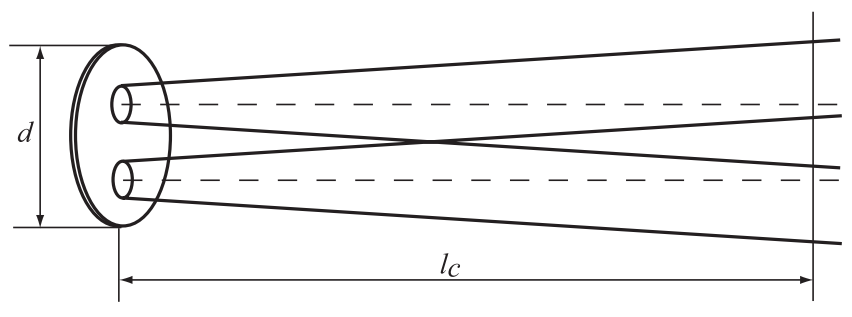

FIG. 4. Scheme of URW's propagation.

of the focused URW beam in the crystal (typically $\sim 0.1 \mathrm{~mm}$ ).

6. The electron bunch spacing in storage rings is much bigger than the bunch length. The same time structure of the OPA must take advantage on this circumstance.

7. OPA amplifies linearly polarized radiation. That is why the circular polarization of the URWs (if helical undulator is used) in our case must be transformed into the linear polarized one before it will be injected in the OPA and then back to be used in the kicker undulator. The usual quarter wave plate can be used for this purpose in the simplest case. Planar undulators can be used as well. Reflective optics can be used for dispersion-free undulator light propagation.

8. OPA consists of one or more nonlinear crystals (NLC) and a laser system for NLC pumping. The system consists of a master oscillator and a picking device to reduce the frequency of master oscillator down to that of bunches in the storage ring $f_{r}$. When the optical pulse length is equal or greater than the bunch length, the master oscillator length is to be set at the harmonic of $f_{r}$ precisely. In the case of laser pulse length substantially shorter than the bunch length, in some cases, a new master oscillator design can be used. The main idea is the periodic change of cavity length in the master oscillator. If the length exceeds the length of a cavity tuned to $f_{r}$, an optical pulse gradually (cycle by cycle) shifts to the trailing edge of a bunch. On the other hand, if the length is less than the length of a cavity tuned to $f_{r}$, an optical pulse gradually shifts to the leading edge of a bunch.

\section{USEFUL EXPRESSIONS FROM THE THEORY OF CYCLIC ACCELERATORS}

The equilibrium value of relative energy spread of the electron beam in the isomagnetic lattice of the storage ring determined by synchrotron radiation (SR) is described by expression

$$
\frac{\sigma_{E}^{\mathrm{eq}, \mathrm{SR}}}{E}=\sqrt{\frac{55 \Lambda \gamma^{2}}{32 \sqrt{3} R_{s} \widetilde{\Im}_{s}}} \simeq 6.2 \times 10^{-6} \frac{\gamma}{\sqrt{R_{s} \mathfrak{\Im}_{s}}},
$$

where $\Lambda_{c}=\hbar / m c \simeq 3.86 \times 10^{-11} \mathrm{~cm}, R_{s}$ is the equilibrium bending radius of the synchronous electron, in the smooth approximation $\mathfrak{\Im}_{s}=4-\alpha_{c} \bar{R}_{s} / R_{s}$ is a coefficient 
determined by the magnetic lattice $\left(0<\Im_{s}<3\right), \alpha_{c}$ is the momentum compaction factor [16].

For small synchrotron oscillations the equilibrium length of the electron bunch is

$$
\sigma_{s}^{\mathrm{eq}, \mathrm{SR}}=\frac{\alpha_{c} c}{\Omega} \frac{\sigma_{E}^{\mathrm{eq}, \mathrm{SR}}}{E}
$$

where $\Omega=\omega_{\text {rev }} \sqrt{h \alpha_{c} e V_{0} \cos \phi_{s} / 2 \pi E_{s}}$ is the synchrotron frequency, $\omega_{\text {rev }}=2 \pi f, h$ is an accelerating rf voltage harmonic order.

The equilibrium radial synchrotron and betatron beam dimensions are

$$
\begin{aligned}
\sigma_{x_{\eta}}^{\mathrm{eq}, \mathrm{SR}} & =\eta_{x} \frac{\sigma_{E}^{\mathrm{eq}, \mathrm{SR}}}{E}, \\
\sigma_{x}^{\mathrm{eq}, \mathrm{SR}} & =\gamma \sqrt{\frac{55 \sqrt{3} \Lambda \overline{R_{s}}}{96} \frac{F}{\Im_{x}}}=6.2 \times 10^{-6} \gamma \sqrt{\frac{\overline{R_{s}} F}{\Im_{x}}},
\end{aligned}
$$

where in the smooth approximation $\mathfrak{\Im}_{x}=\alpha_{c} \bar{R}_{s} / R_{s}-1$ is a coefficient determined by the magnetic lattice $\left(\Im_{x}+\right.$ $\left.\mathfrak{\Im}_{s}=3\right), F \sim 1$ is a coefficient $[16,17]$.

The damping time in the storage ring determined be synchrotron radiation in the bending magnets and comes to the following values $\left(\mathfrak{I}_{z}=1\right)$ :

$$
\tau_{x, z, s}^{\mathrm{SR}}=\frac{2 E}{P_{\mathrm{SR}, s} \widetilde{\Im}_{x, z, s}}=\frac{3 R_{s} \overline{R_{s}}}{c r_{e} \gamma^{3} \mathfrak{\Im}_{x, z, s}}=355 \frac{R_{s} \overline{R_{s}}}{\gamma^{3} \mathfrak{\Im}_{x, z, s}}[\mathrm{sec}]
$$

The maximum deviation of the energy from its synchronous one is

$$
\frac{(\Delta E)_{\mathrm{sep}}}{E}= \pm \beta \sqrt{\frac{e V_{0}}{\pi h \eta_{c} E}\left[\left(\pi-2 \phi_{s}\right) \sin \phi_{s}-2 \cos \phi_{s}\right]}
$$

where $\eta_{c}=\alpha_{c}-1 / \gamma^{2}$ is the slippage factor of the ring.

For the ordinary storage ring lattice (without local isochronous bend or bypass between undulators), the natural local slippage factor

$$
\eta_{c, l} \simeq L_{p, k} \eta_{c} / C
$$

\section{EXAMPLES}

Below we consider two examples of one-dimensional EOC in the bucket of an electron beam in the transverse $x$-plane in $2.5 \mathrm{GeV}$ strong focusing storage ring Siberia-2 (Kurchatov Institute Atomic Energy, Moscow) [18]. The magnetic lattice of the ring has separate functions. It consists of six mirror-symmetrical superperiods, each containing an achromatic bend and two $3 \mathrm{~m}$ long straight sections. For functionality, the half of the superperiod is arranged with two sections. The first one, comprising the quadru-

TABLE I. Parameters of the ring.

\begin{tabular}{ll}
\hline \hline The maximal energy of the storage ring & $E_{\max }=2.5 \mathrm{GeV}$ \\
The energy for the experiment & $E_{\exp }=100 \mathrm{MeV}$ \\
Relativistic factor for the experiment & $\gamma \cong 200$ \\
Circumference & $C=124.13 \mathrm{~m}$ \\
Bending radius & $R=490.54 \mathrm{~cm}$ \\
Average radius & $\bar{R}=1962 \mathrm{~cm}$ \\
Revolution frequency & $f=2.42 \times 10^{6} \mathrm{~Hz}$ \\
Harmonic number & $h=75$ \\
rf frequency & $f_{\text {rf }}=181.14 \mathrm{MHz}$ \\
Energy loss determined by SR & $P_{\gamma, S \mathrm{R}} / f=1.89 \mathrm{eV} / \mathrm{turn}$ \\
The amplitude of the accelerating voltage at $E_{\text {exp }}$ & $V_{0}=73 \mathrm{~V}$ \\
The synchronous phase & $\varphi_{s} \simeq 0.026$ \\
Radial tune & $v_{x}=7.731$ \\
Vertical tune & $v_{z}=7.745$ \\
Momentum compaction factor & $\alpha_{c}=7.6 \times 10^{-3}$ \\
Dispersion function at the pickup and the kicker undulator & $\eta_{x}=80 \mathrm{~cm}$ \\
Radial beta function in pickup undulator & $\beta_{x}=17 \mathrm{~m}$ \\
Radial beta function in kicker undulator & $\beta_{x}=1.7 \mathrm{~m}$ \\
Vertical beta function & $\beta_{z}=6 \mathrm{~m}$ \\
Patrician coefficients $\Im_{z}, \Im_{x}, \Im_{s}$ & $1,0.97,2.03$ \\
Damping times by SR at $E_{\text {exp }} \tau_{z}, \tau_{x}, \tau_{s}$ & $43.1 ; 44.4 ; 21.23 \mathrm{sec}$ \\
The length of the period of betatron oscillations & $\lambda_{x, \text { bet }}=16.06 \mathrm{~m}$ \\
Slippage factor of the ring & $\eta_{c}=\alpha_{c}$ \\
Local slippage factor of the ring & $\eta_{c, l}=0.58 \cdot \alpha_{c}$ \\
Frequency of synchrotron oscillations at $E_{\text {exp }}=100 \mathrm{MeV}$ & $\Omega=1.6 \times 10^{-3} \mathrm{f}$ \\
\hline \hline
\end{tabular}


TABLE II. Initial parameters of the electron beam in the ring.

\begin{tabular}{ll}
\hline \hline Number of electrons at the orbit & $N_{e, \Sigma}=5 \times 10^{4}$, \\
Number of electron bunches being cooled & 1 \\
Relative energy spread & $\sigma_{E, 0} / E=3.94 \times 10^{-5}$, \\
Betatron beam size at pickup undulator $\left(\beta_{x}=32 \mathrm{~m}\right)$ & $\sigma_{x, 0}=4 \mathrm{~mm}$, \\
Betatron beam size at kicker undulator $\left(\beta_{x}=2 \mathrm{~m}\right)$ & $\sigma_{x, 0}=1 \mathrm{~mm}$, \\
Dispersion beam size & $\sigma_{x_{\eta}, 0}=3.15 \times 10^{-2} \mathrm{~mm}$, \\
Total beam size at pickup undulator & $\sigma_{x, \text { tot }}=\sqrt{\left(\sigma_{x_{\eta}, 0}\right)^{2}+\left(\sigma_{x, 0}\right)^{2}}=4 \mathrm{~mm}$, \\
The length of the electron bunch & $\sigma_{s, 0}=2.32 \mathrm{~cm}$, \\
\hline \hline
\end{tabular}

poles $\mathrm{F}, \mathrm{D}, \mathrm{F}$, and two bending magnets is responsible for the achromatic bend and high $\boldsymbol{\beta}_{x}, \boldsymbol{\beta}_{y}$ functions in the undulator straight section. The second part, comprising quadrupoles D, F, D, and a dispersion-free straight section, allows one to change the betatron tunes, without disturbing the achromatic bend. The main parameters of the ring, the electron beam in the ring, pickup and kicker undulators, and OPA are presented in Tables I, II, III, and IV.

We accept the distance between pickup and the kicker undulator along the synchronous orbit $L_{p, k}=72.27 \mathrm{~m}$ $\left(\psi_{x}^{\text {bet }}=9 \pi, k_{p, k}=4\right)$. It corresponds to the installation of undulators in the first and seventh straight sections which are located at a distance $72.38 \mathrm{~m}$ (we count off pickup undulator). The second kicker undulator can be located on the same distance from the first one. The optical line is tuned in such a way that electrons are decelerated in the first kicker undulator and accelerated in the second one.

A single electron bunch is used in the storage ring at the energy $100 \mathrm{MeV}$ in the experiment. The beam is cooled by synchrotron radiation damping (see Sec. V). The energy spread and the beam size acquire equilibrium values in $\sim 40$ seconds after its injection in the ring (see Table I). The equilibrium energy spread is equal to $\sigma_{E}^{\mathrm{eq}, \mathrm{SR}} / E=$ $3.94 \times 10^{-5}$, the length of the bunch $\sigma_{s}^{\text {eq, SR }}=2.32 \mathrm{~cm}$ at the amplitude of the accelerating voltage $V_{0}=73 \mathrm{~V}$, the synchronous voltage $V_{s}=1.89 \mathrm{~V}$, the radial emittance

TABLE III. Parameters of pickup and kicker undulators.

\begin{tabular}{ll}
\hline \hline Magnetic field strength & $\sqrt{\bar{B}^{2}}=1338 \mathrm{Gs}$, \\
Undulator period & $\lambda_{u}=8 \mathrm{~cm}$, \\
Number of periods & $M=30$, \\
Deflection parameter & $K=1$. \\
\hline \hline
\end{tabular}

$\epsilon_{x}^{\mathrm{eq}, \mathrm{SR}}=1.25 \times 10^{-6} E^{2}[\mathrm{GeV}]=1.25 \times 10^{-8} \mathrm{~cm} \mathrm{rad}$, the radial betatron beam dimension at pickup undulator $\sigma_{x}^{\mathrm{eq}, \mathrm{SR}}=4.61 \times 10^{-2} \mathrm{~mm}$. The energy spread of the beam limited by the separatrix is $\Delta E_{\mathrm{sep}} / E=3.3 \times 10^{-4}$. Following synchrotron radiation damping, the amplitudes of radial betatron oscillations $\sigma_{x, 0}$ are artificially excited to be suitable for resolution of the electron beam in the experiment with EOC (see Table II). The amplitudes of synchrotron oscillations must stay damped to work with short electron bunches and short duration of amplification OPAs. We took the number of electrons at the orbit $N_{e}=$ $5 \times 10^{4}$. In this case the number of electrons in the URW sample is $N_{e, s}=129.5$, the number of extraneous photons in the sample is $N_{\mathrm{ph}, \Sigma}=2.48$ for the case of one noise photon at the OPA front end.

The number of electrons in the bunch is enough to detect them in the experiment and not so high to neglect intrabeam scattering.

The URWs have the number of photons emitted in the pickup undulator (see Table III) $\left.N_{\mathrm{ph}}^{\mathrm{cl}}\right|_{K=1}=1.15 \times 10^{-2}$ per electron in the frequency and angular ranges (3) suitable for cooling. The limiting amplitude of betatron oscillations (14) is $A_{x, \lim }=1.8 \mathrm{~mm}$. The electric field strength of the nonamplified URW in the kicker undulator is $E_{w}^{\mathrm{cl}} \cong$ $2.06 \times 10^{-3} \mathrm{~V} / \mathrm{cm}$. The maximal power loss for the electron passing through one kicker undulator together with its amplified URW comes to $P_{\text {loss }}^{\max }=1.02 \times 10^{5} \mathrm{eV} / \mathrm{sec}$ if the amplification gain of OPA is $\alpha_{\text {ampl }}=2.5 \times 10^{4}$ (see Tables II and IV). This power loss corresponds to the maximal energy jumps $\Delta E_{\text {loss }}^{\max }=3.66 \mathrm{eV}$ and the maximal average energy loss per turn $\bar{\Delta} E_{\text {loss }}^{\text {turn }}=0.042 \mathrm{eV} /$ turn. The jump of the closed orbits is $\delta x_{\eta}^{1}=2.92 \times 10^{-6} \mathrm{~cm}$. In this storage ring the natural local slippage factor (34) is

TABLE IV. Optical parametric Amplifier.

\begin{tabular}{ll}
\hline \hline Number of optical parametric amplifiers & 2 \\
Total gain & $\begin{array}{l}\alpha_{\text {ampl }}=10^{7} \\
\text { The wavelength of amplified URWs }\end{array}$ \\
The characteristic URW waist size & $\sigma_{1, \text { min }}=2 \times 10^{-4} \mathrm{~cm}$ \\
The URW beam waist size & $\sigma_{w}=2 \mathrm{~mm}$ \\
The duration of the amplification time of the OPA & $15 \mathrm{msec}\left(l_{\mathrm{ampl}}=4.5 \mathrm{~mm}\right)$ \\
The frequency of the amplified cycles & $f_{\mathrm{ampl}}=f=2.42 \times 10^{6} \mathrm{~Hz}$ \\
\hline
\end{tabular}


$\eta_{c, l} \simeq \alpha_{c} L_{p, k} / C=4.45 \times 10^{-3}$, the energy gap (18) is $\delta E_{\text {gap }}=0.62 \mathrm{keV}$, and the equilibrium energy spread (12) is $\sigma_{E \text {,eq }}^{\mathrm{EOC}}=0.28 \mathrm{keV}$.

In the variants of the example considered below the optical system resolution of electron beam, according to (28), is $\delta x_{\text {res }}=1.9 \mathrm{~mm}$ at $\lambda_{1, \min }=2 \times 10^{-4} \mathrm{~cm}(\hbar \omega \simeq$ $0.5 \mathrm{eV}), M \lambda_{u}=240 \mathrm{~cm}$. It yields that effective EOC in this case is possible if the beam under cooling has a total size in the pickup undulator $\sigma_{x \text {,tot }}>2.0 \mathrm{~mm}$. The situation could be better if we had effective OPA using the wavelength $\lambda_{1, \min } \simeq 3 \times 10^{-5} \mathrm{~cm}$, i.e., about one order less. The shorter undulator can be used as well to improve the electron beam resolution.

We accepted the initial energy spread $\sigma_{E, 0}=\sigma_{E}^{\text {eq,SR }}=$ $3.94 \times 10^{3} \mathrm{keV}$, the dispersion beam size $\sigma_{x_{\eta}, 0}=3.15 \times$ $10^{-2} \mathrm{~mm}$, the length of the electron bunch $\sigma_{s, 0}=$ $2 \sigma_{s}^{\mathrm{eq}, \mathrm{SR}}=4.64 \mathrm{~cm}$, its transverse size at pickup undulator $\sigma_{x, 0}=4 \mathrm{~mm}$, the radial betatron beam size in kicker undulator $\sigma_{x, 0}=1 \mathrm{~mm}$, and the URW beam size $\sigma_{w, b}=$ $2 \mathrm{~mm}$. The accepted energy spread $\sigma_{E, 0}$ of the electron bunch was chosen small in order to have as small as possible the length of this bunch and, according to (25), the damping time. Moreover, it goes into $2 M+1$ energy gaps in the ring.

Below we consider EOC in the bucket in the transverse plane. In this case the dispersion beam size is much less than the special resolution of the optical system $\sigma_{x_{\eta}, 0} \ll$ $\delta x_{\text {res }}$ and that is why there is no selection of electrons in the longitudinal plane (see variants 1, 6 in Sec. III). Electrons in the kicker undulator change their energy up or down with equal probability both at positive and negative deviations of their closed orbit from the synchronous one. That is why the energy spread of the beam is not changed in the first approximation for one kicker undulator. Using two kicker undulators permits one to neglect the multiple processes of excitation of synchrotron oscillations in the second approximation. The amplitudes of betatron oscillations of electrons depending on the initial phases $\varphi_{\text {in }}$ are decreased (increased) at every loss (gain) of their energy until they accept equilibrium values $\varphi_{m}=\pi / 2+2 \pi m$. Using variable time delay will permit EOC.

Case 1.-The local slippage factor $\boldsymbol{\eta}_{c, l}=0$, amplitudes of betatron oscillations are higher than limiting ones (14), the rf accelerating system is switched on, a moving screen opens the way only to URWs emitted by electrons with the energy higher than the synchronous one. In this case the difference in the propagation time of the URW and the traveling time $T_{p, k}$ of the electron between pickup and kicker undulators is $\Delta t=$ const for given amplitudes. Hence, we can install the initial phase $\varphi_{\text {in }}=\varphi_{\text {in,bet }} \simeq \pi$ for all electrons with zero amplitudes of betatron oscillations. The rest of the electrons will have initial phases proportional to their amplitudes. The electron phases will vary continuously with the change of the electron energy and amplitude to the level corresponding to their equilibrium values $\varphi_{m}=\pi / 2+2 \pi m$. The variable in time optical delay line is used to decrease the length of the light pass-way between undulators and equilibrium phases during the cooling cycle and to move the phases to the phase $\left.\varphi_{m}\right|_{m=0}=\pi / 2$ and amplitudes to $A_{n}=0$.

For the parameters presented above and two kicker undulators the cooling time for the transverse coordinate, according to (10), comes to $\tau_{x, \mathrm{EOC}}=3.7 \mathrm{sec}$ at the laser amplification length $l_{\mathrm{ampl}}^{\text {laser }}=\sigma_{s, 0}=2 \sigma_{s}^{\mathrm{eq}, \mathrm{SR}}=4.64 \mathrm{~mm}$. SR damping time is much bigger ( $\sim 40 \mathrm{sec})$.

Necessary conditions for selection of electrons must be created: high beta function in the pickup undulator (to increase the transverse dimensions of the bunch for selection of electrons at positive deviations from closed orbit), the isochronous bend between undulators for zero local slippage factor. We believe that the lattice of the ring is flexible enough to be changed in necessary limits by analogy with those presented in [19].

Case 2. - The local slippage factor $\eta_{c, l} \neq 0$, amplitudes of betatron oscillations are higher than limiting ones (14), $\sigma_{E \text {,eq }}^{\mathrm{EOC}} \ll \delta E_{\text {gap }}$, the moving screen opens the way only to URWs emitted by electrons with the energy higher than the synchronous one $E_{s}$, optical system install the initial delay time of the URWs so that they interact with electrons at the energies $E \geq E_{s}$ and change the delay time of the URWs to move the energies of electrons to the energy $E_{s}$. Then electrons with smaller energy are captured increasingly in the regions of the energy layers $E_{m}$ and lose their energy and amplitudes of betatron oscillations until their energy takes equilibrium one and amplitudes are decreased. Cooling takes place according to the scheme of the EOC. This process can be repeated.

The conditions (23) and (24) or $l_{\mathrm{ampl}}^{\text {laser }}<l_{\mathrm{ampl}, 1,2}$ limit the length of the laser URWs by the values $l_{\mathrm{ampl}, 1}=1.69 \mathrm{~cm}$, $l_{\mathrm{ampl}, 2}=0.32 \mathrm{~cm}$. The accepted laser amplification length $l_{\text {ampl }}^{\text {laser }}=3.2 \mathrm{~mm}$ is enough to satisfy these conditions. The damping time for radial betatron oscillations, according to (25), is $\tau_{x, \text { EOC }} \simeq 5.4 \mathrm{sec}$ if two kicker undulators are used.

\section{CONCLUSIONS}

We have shown in this paper that a test of EOC is possible in the $2.5 \mathrm{GeV}$ electron strong focusing storage ring tuned down to the energy $\sim 100 \mathrm{MeV}$. The electron beam can be cooled in the transverse direction. The damping time is much less than one determined by synchrotron radiation. So the EOC can be identified by the change of the damping rate of the electron beam. A variant of cooling is found, which permits one to avoid any changes in the existing lattice of the ring (for production of isochronous bend, bypass). It can work for existing ion storage rings as well (see Appendix B). Three short undulators in this variant installed in the storage ring have rather long periods and weak fields. They can be manufactured at low cost. 
The cooling of a relatively small number of electrons (one bunch, $N_{e, \Sigma}=5 \times 10^{4}$ ) is considered in this proposal in an attempt to avoid strong influence of the nonsynchronous URWs on the equilibrium energy spread of the beam. The intrabeam scattering effects could be overcome as well. Optical amplifier suitable for the EOC-so-called optical parametric amplifier-suggested as a baseline of the experiment, must have moderate gain and power. We have chosen the wavelength of the OPA equal $2 \mathrm{mkm}$ because in this case OPA is pumped directly by a commercially available highly efficient picosecond solid state laser. At the present time, the OPAs having amplification gain $\sim 10^{8}$ and the power $>1 \mathrm{~W}$ fully satisfy requirements for this experiment (see Appendix C). Usage of OPAs with shorter wavelength will permit one to increase the spatial resolution by the optical system and the degree of cooling of the beam.

We have predicted that the maximum rate of energy loss for electrons in the fields of the kicker undulator and amplified URW calculated in the framework of classical electrodynamics is $\sqrt{N_{\mathrm{ph}}} \simeq 9.3$ times less than one taking into account the quantum nature of the photon emission in undulators. Quantum aspects of the beam physics will be checked in the proposed test experiment. It is suggested that the scheme based on optical line with variable delay time will be tested as well.

Similar considerations can be made for any damping ring in service.

Using a movable screen and an optical line with a variable time delay in the OSC leads to new opportunities of enhanced cooling.

\section{ACKNOWLEDGMENTS}

The authors thank A. V. Vinogradov and Yu. Ya. Maslova for useful discussions. This work was supported by RFBR under Grants No. 05-02-17162a, No. 05-0217448a, by the Program of Fundamental Research of RAS, subprogram "Laser systems", and NSF Grant No. PHY-0202078.

\section{APPENDIX A}

Spectral-angular distribution of the UR energy emitted by the relativistic electron in the pickup undulator on the harmonic $n$ is

$$
\frac{\partial^{2} E_{n}}{\partial \omega \partial o}=\frac{M}{\omega_{1}} \frac{\partial E_{n}}{\partial o} \sin c^{2} \sigma_{n}(\omega, \vartheta)
$$

where $\partial E_{n} / \partial o$ is the angular distribution of the energy of the UR emitted in the unit solid angle $d o$ at the angle $\theta$ to its axis, $\sin c \sigma_{n}=\sin \sigma_{n} / \sigma_{n}, \sigma_{n}=\pi n M\left(\omega-\omega_{n}\right) / \omega_{n}$, $\omega_{n}=n \omega_{1}$ is the frequency of the $n$th harmonic of UR [20-22]. For the helical undulator

$$
\begin{aligned}
\frac{\partial E_{n}}{\partial o} & =\frac{e^{2} M n^{2} \omega_{1}^{3}(\vartheta) \beta_{\perp}^{2} F_{n}(K, \vartheta)}{c \Omega^{2}} \\
& =\frac{6 E_{\mathrm{tot}} \gamma^{2}}{\pi} \frac{n^{2} F_{n}(K, \vartheta)}{\left(1+K^{2}+\vartheta^{2}\right)^{3}}
\end{aligned}
$$

$F_{n}(K, \vartheta)=J_{n}^{\prime 2}(n \chi)+\left(\frac{1+K^{2}-\vartheta^{2}}{2 K \vartheta}\right)^{2} J_{n}^{2}(n \chi), \quad E_{\mathrm{tot}}=$ $4 \pi e^{2} \Omega M \gamma^{2} K^{2} / 3 c, \Omega=2 \pi c / \lambda_{u}, J_{n}, J_{n}^{\prime}$ is the Bessel function and its derivative, $\chi=2 K \vartheta /\left(1+K^{2}+\vartheta^{2}\right)<$ $1, \beta_{\perp}=K / \gamma$. The number of photons emitted in the undulator on the harmonic $n$ in the solid angle $d o=$ $\pi d \vartheta^{2} / \gamma^{2}$ and frequency band $d \omega$ is determined by the relation

$$
\begin{aligned}
N_{\mathrm{ph}, n}= & \frac{\alpha n^{2} M^{2} K^{2} \lambda_{u}}{\pi c} \iint \frac{F_{n}(K, \vartheta)}{\left(1+K^{2}+\vartheta^{2}\right)^{2}} \\
& \times \sin c^{2} \sigma_{n}(\omega, \vartheta) d \omega d o .
\end{aligned}
$$

If the considered frequency band $\Delta \omega / \omega \ll 1 / M \ll 1$, then we can neglect the angular dependence of the first multiplier and the frequency dependence of the value $\sin c \sigma_{n}$ in (A2). In this case the value $\sin c \sigma_{n}$ determines the range of angles of the UR (3). The increasing of the frequency band will lead to the increase of the angular range. Taking the frequency band $\Delta \omega$ out of the integral and taking into account that $d \vartheta^{2}=\left(2 \Omega \gamma^{2} / \pi M \omega_{1}\right) d \sigma_{n}$, $\sin c \sigma_{n}=\pi \delta\left(\sigma_{n}\right)$, we can transform (A2) to (6) for $\Delta \omega / \omega=1 / 2 M \vartheta=0$, and $n=1$.

\section{APPENDIX B}

Below we investigate the possibility of lead ions cooling $(Z=82)$ in the storage ring LHC based on using version 5 (Sec. III) of EOC. We take example 2 considered in [7]. In this case the energy $E=1.85 \times 10^{14} \mathrm{eV},(\gamma=953)$ the slippage factor of the ring $\eta_{c} \simeq \alpha_{c}=3.23 \times 10^{-4}$, the amplitude of the $\mathrm{rf}$ accelerating voltage $V_{0}=16 \mathrm{MV}$, the RMS bunch length $8 \mathrm{~cm}$, rf frequency $f_{\text {rf }}=400 \mathrm{MHz}$, synchrotron frequency $\Omega=23 \mathrm{~Hz}$, circumference $C=$ $2665888.3 \mathrm{~cm}$, harmonic order $h=35640$, rms relative energy spread $\sigma_{E, 0} / E=1.1 \times 10^{-4}, \quad \sigma_{E, 0}=2.04 \times$ $10^{10} \mathrm{eV}, \lambda_{x, \text { bet }}=415 \mathrm{~m}$, rf bucket half-height $\Delta E_{\text {sep }} / E=$ $4.43 \times 10^{-4}, \Delta E_{\text {sep }}=8.19 \times 10^{10} \mathrm{eV}$. We take the distance between pickup and kicker undulators $L_{p, k}=$ $1453 \mathrm{~m}(k=3)$, synchrotron radiation energy loss per ion per turn $P_{\mathrm{SR}, s} / f=1.2 \times 10^{4} \mathrm{eV}$.

For the parameters of the undulators [7], the energy loss per turn is $\Delta E_{\text {loss }}^{\max }=3 \times 10^{5} \mathrm{eV}, \sigma_{E, 0} / M=1.7 \times 10^{9} \mathrm{eV}$ $(M=12)$, the gap between equilibrium energy positions is $\delta E_{\text {gap }}=1.97 \times 10^{8} \mathrm{eV}$ at $\lambda_{1, \text { min }}=5 \times 10^{-5} \mathrm{~cm}, N_{\mathrm{ph}}^{\mathrm{cl}}=$ $6.29, \sigma_{E \text {,eq }}^{\mathrm{EOC}}=4.7 \times 10^{6} \mathrm{eV}$ at the number of ions in the bunch $N_{i}=10^{6}, N_{i, s}=132$ at the length of the bunch $0.95 \mathrm{~cm}$. It follows that $\sigma_{E \text {,eq }}^{\mathrm{EOC}} \ll \delta E_{\text {gap }}$ and that the condition (19) is satisfied. In the case of ions the equation (22) must include $e Z \cdot V_{0}$ instead of $e V_{0}$. In this example $\delta E_{\text {loss }}^{\max } / e Z V_{0}=2.2 \times 10^{-4}$. It follows the laser amplifica- 
tion length $l_{\mathrm{ampl}, 2}=2.78 \mathrm{~mm}$. By this choice the condition (22) is satisfied as well. To cool the ion beam in the transverse plane and to keep the magnetic lattice unchanged, one pickup and two kicker undulators must be used.

\section{APPENDIX C}

The principle of OPA is quite simple $[23,24]$ : in a suitable nonlinear crystal, a high frequency and high intensity beam (the pump beam, at frequency $\omega_{p}$ ) amplifies a lower frequency, lower intensity beam (the signal beam, at frequency $\omega_{s}$ ); in addition, a third beam (the idler beam, at frequency $\omega_{i}$, with $\omega_{i}<\omega_{s}<\omega_{p}$ ) is generated. (In the OPA process, signal and idler beams play an interchangeable role, we assume that the signal is at higher frequency, i.e., $\omega_{s}>\omega_{i}$.) In the interaction, energy conservation

$$
\hbar \omega_{p}=\hbar \omega_{s}+\hbar \omega_{i}
$$

is satisfied; for the interaction to be efficient, also the momentum conservation (or phase-matching) condition

$$
\hbar \mathbf{k}_{p}=\hbar \mathbf{k}_{s}+\hbar \mathbf{k}_{i}
$$

where $\mathbf{k}_{p}, \mathbf{k}_{s}$, and $\mathbf{k}_{i}$ are the wave vectors of pump, signal, and idler, respectively, must be fulfilled. The signal frequency to be amplified can vary in principle from $\omega_{p} / 2$ (the so-called degeneracy condition) to $\omega_{p}$, and correspondingly the idler varies from $\omega_{p} / 2$ to 0 ; at degeneracy, signal and idler have the same frequency. In summary, the OPA process transfers energy from a high-power, fixed frequency pump beam to a low-power, variable frequency signal beam, thereby generating also a third idler beam.

The signal and idler group velocities $v_{s}$ and $v_{i}(\mathrm{GVM}-$ group velocity mismatch) determine the phase-matching bandwidth for the parametric amplification process. Let us assume that perfect phase matching is achieved for a given signal frequency $\omega_{s}$ (and for the corresponding idler frequency $\omega_{i}=\omega_{p}-\omega_{s}$ ). If the signal frequency increases to $\omega_{s}+\Delta \omega$, by energy conservation the idler frequency decreases to $\omega_{i}-\Delta \omega$. The wave vector mismatch can then be approximated to the first order as

$$
\Delta k \cong-\frac{\partial k_{s}}{\partial \omega_{s}} \Delta \omega+\frac{\partial k_{i}}{\partial \omega_{i}} \Delta \omega=\left(\frac{1}{\nu_{g i}}-\frac{1}{\nu_{g s}}\right) \Delta \omega .
$$

The gain bandwidth of an OPA can be estimated using the analytical solution of the coupled wave equations in the slowly varying envelope approximation and assuming flat top spatial and temporal profiles and no pump depletion. The intensity gain $(G)$ and phase $(\varphi)$ of the amplified signal beam are given in [25] by

$$
\begin{aligned}
G & =1+(\gamma L)^{2}\left(\frac{\sinh B}{B}\right)^{2}, \\
\varphi & =\tan ^{-1} \frac{B \sin A \cosh B-A \cos A \sinh B}{B \cos A \cosh B-A \sin A \sinh B},
\end{aligned}
$$

where $A=\Delta k L / 2, B=\sqrt{(\gamma L)^{2}-(\Delta k L / 2)^{2}}$, and $\gamma=$ gain coefficient $=4 \pi d_{\mathrm{eff}} \sqrt{I_{p} / 2 \varepsilon_{0} n_{p} n_{s} n_{i} c \lambda_{s} \lambda_{i}}, \quad \Delta k L=$ phase mismatch $=\left(\mathbf{k}_{p}-\mathbf{k}_{s}-\mathbf{k}_{i}\right) L$, where $L$ is the length of amplifier, $d_{\text {eff }}$ is the effective nonlinear coefficient, $I_{p}$ is the pumping intensity.

The full width at half maximum (FWHM) phasematching bandwidth can then be calculated within the large-gain approximation as

$$
\Delta \nu \cong \frac{2(\ln 2)^{1 / 2}}{\pi}\left(\frac{\gamma}{L}\right)^{1 / 2} \frac{1}{\left|\frac{1}{\nu_{g s}}-\frac{1}{\nu_{g i}}\right|} .
$$

The large GVM between signal and idler waves dramatically decreases the phase-matching bandwidth; large-gain bandwidth can be expected when the OPA approaches degeneracy $\left(\omega_{s} \rightarrow \omega_{i}\right)$ in type I phase matching or in the case of group velocity matching between signal and idler $\left(\nu_{g s}=\nu_{g i}\right)$. Obviously, in this case Eq. (C2) loses validity and the phase mismatch $\Delta k$ must be expanded to the second order, giving

$$
\Delta \nu \cong 2 \frac{(\ln 2)^{1 / 4}}{\pi}\left(\frac{\gamma}{L}\right)^{1 / 4} \frac{1}{\left|\frac{\partial^{2} k_{s}}{\partial \omega_{s}^{2}}+\frac{\partial^{2} k_{i}}{\partial \omega_{i}^{2}}\right|} .
$$

For the case of perfect phase matching $(\Delta k=0, B=\gamma L)$ and in the large-gain approximation $(\gamma L \gg 1)$, Eqs. (4) and (C1) simplify to

$$
I_{s}(L) \cong \frac{1}{4} I_{s 0} \exp (2 \gamma L), \quad I_{i}(L) \cong \frac{\omega_{i}}{4 \omega_{s}} I_{s 0} \exp (2 \gamma L) .
$$

Note that the ratio of signal and idler intensities is such that an equal number of signal and idler photons are generated. Equations (C3) allow defining a parametric gain as

$$
G \cong \frac{1}{4} \exp (2 \gamma L) \cong \frac{1}{4} \exp \left(8 L \pi d_{\mathrm{eff}} \sqrt{I_{p} / 2 \varepsilon_{0} n_{p} n_{s} n_{i} c \lambda_{s} \lambda_{i}}\right)
$$

growing exponentially with the crystal length $L$ and with the nonlinear coefficient $\gamma$.

The noise (amplified self-emission) power of the optical amplifier is determined by the expression

$$
P_{n}=P_{n, 0} G_{0}
$$

where $P_{n, 0}$ is the noise power at the amplifier front end, $G_{0}$ is the gain of the amplifier.

If the noise power corresponds to one-photon/mode at the amplifier front end then $P_{n, 0}=\hbar \omega_{1 \max } / \tau_{\text {coh }}$ [26,27], where in our case $\tau_{\text {coh }}=2 M T$ is the coherence length, $T=\lambda_{1 \min } / c$. 


\section{Example 1: Infrared OPA based on MgO periodically poled lithium niobate}

In recent years the development of periodically poled nonlinear materials has enhanced the flexibility and performance of OPAs. In the case of well-studied periodically poled lithium niobate (PPLN), one can get access to the material's highest effective nonlinearity as well as retain generous flexibility in phase-matching parameters and nonlinear interaction lengths. PPLN well match with commercially available highly efficient diode pumped picosecond solid state lasers. For wideband optical parametric amplification, we will use MgO: PPLN with a poling period of 31.1 (31.2) $\mu \mathrm{m}$, which has high damage threshold and high nonlinear coefficient $16 \mathrm{pm} / \mathrm{V}$ [28] (17 pm/V [29]). Operation near the degeneracy wavelength of $2.128 \mu \mathrm{m}$ reduces thermal-lens effect because the signal and the idler wavelengths fall within the highest transparency range of lithium niobate. To avoid photorefractive damage, thick $(\sim 1-2 \mathrm{~mm})$ the PPLN crystal will be kept at elevated temperatures $150^{\circ} \mathrm{C}$ [30].

For the signal wavelength $\lambda_{s}=2 \mu \mathrm{m}, n_{s} \approx n_{p}=2.1$, and the gain, according to formula (C4) comes to

$$
G \cong \frac{1}{4} \exp \left[3\left(\frac{I_{p}}{\mathrm{GW} / \mathrm{cm}^{2}}\right)^{1 / 2} \frac{l}{\mathrm{~mm}}\right]
$$

For $G=10^{7}, I_{p}=1 \mathrm{GW} / \mathrm{cm}^{2}, l=5.8 \mathrm{~mm}$. We propose the two-stage crystal amplifier $\left(l_{1}=l_{2}=3.5 \mathrm{~mm}\right)$. OPA amplifies linearly polarized radiation. That is why the circular polarization of the URWs (if helical undulator is used) in our case must be transformed into the linear polarized one before it will be injected in the OPA and then back to be used in the kicker undulator. The usual quarter wave plate can be used for this purpose in the simplest case. Planar undulators can be used as well. Reflective optics can be used for dispersion-free undulator light propagation.

\section{Example 2: Near-ultraviolet OPA based on Beta-Barium Borate (b-BaB $\left.\mathbf{B}_{2} \mathrm{O}_{4}\right)$}

Usage of OPAs with shorter than considered above wavelength will permit one to increase the spatial resolution by the optical system and the degree of cooling of the beam. Beta-barium borate $\left(\mathrm{b}-\mathrm{BaB}_{2} \mathrm{O}_{4}\right)$, known as $\mathrm{BBO}$, is one of the best candidates for near-ultraviolet OPA pumped by fourth harmonic of a $\mathrm{Nd}$ or $\mathrm{Yb}$ lasers $(\sim 260 \mathrm{~nm})$. For example, femtosecond pulses at $400 \mathrm{~nm}$ were amplified using a noncollinear optical parametric amplifier pumped by picosecond pulses at $267 \mathrm{~nm}$ [31]. The type $\mathrm{I} b-\mathrm{BaB}_{2} \mathrm{O}_{4}$ crystal with $10 \mathrm{~mm}$ length was pumped with $\sim 2.5 \mathrm{GW} / \mathrm{cm}^{2}$. A flat spectral gain exceeding 3500 was achieved in single pass within the available $17 \mathrm{~nm}$ bandwidth of the signal pulse. So a two-stage crystal amplifier $\left(l_{1}=l_{2}=10 \mathrm{~mm}\right)$ and $I_{p}=2.5 \mathrm{GW} / \mathrm{cm}^{2}$ is needed to obtain $G=10^{7}$.
[1] A. M. Sessler, LBL-38278 UC-427, 1996; 31st Workshop: "Crystalline Beams and Related Issues," 1995.

[2] D. Mohl and A. M. Sessler, Nucl. Instrum. Methods Phys. Res., Sect. A 532, 1 (2004).

[3] D. Mohl, CERN Accelerator School Report, CERN No. $87-03,1987$, pp. $453-533$.

[4] E. G. Bessonov, http://arxive.org/abs/physics/0404142.

[5] E. G. Bessonov and A. A. Mikhailichenko, in Proceedings of the 2005 Particle Accelerator Conference, Knoxville, Tennessee, USA, 2005 (http://www.sns.gov/PAC05); http://accelconf.web.cern.ch/accelconf/p05/PAPERS/ TPAT086.PDF.

[6] E. G. Bessonov, A. A. Mikhailichenko, and A. V. Poseryaev, http://arxiv.org/abs/physics/0509196.

[7] E.G. Bessonov, M.V. Gorbunkov, and A.A. Mikhailichenko, in Proceedings of the 2006 European Particle Accelerator Conference, Edinburgh, Scotland, 2006, http://accelconf.web.cern.ch/accelconf/e06/ PAPERS/TUPLS001.PDF; JINST 1, P08005 (2006), http://jinst.sissa.it/, http://ej.iop.org/links/r5pyfrsWE/ sl44atI92xGGY2iAav5vpA/jinst6_08_p08005.pdf .

[8] A. A. Mikhailichenko and M. S. Zolotorev, Phys. Rev. Lett. 71, 4146 (1993).

[9] M. S. Zolotorev and A. A. Zholents, Phys. Rev. E 50, 3087 (1994).

[10] M. Babzich et al., Phys. Rev. ST Accel. Beams 7, 012801 (2004).

[11] E. G. Bessonov, in Proceedings of the 15th International Accelerator Conference on High Energy Accelerators, Hamburg, Germany [Int. J. Mod. Phys. Proc. 1, 138 (1992), Suppl. 2A].

[12] K. Steffen, High Energy Beam Optics (Interscience, New York-London-Sydney, 1964).

[13] A. A. Mikhailichenko, CLNS-98-1539, 1997, Cornell University, p. 14; International Conference on Lasers '97, New Orleans, LA, edited by J. J. Carrol and T. A. Goldman (STS Press, McLean, VA, 1997, pp. 890-897.

[14] D. F. Alferov, Yu. A. Bashmakov, K. A. Belovintsev, E. G. Bessonov, and P. A. Cherenkov, JETP Lett. 26, 385 (1977).

[15] V. I. Alexeev and E. G. Bessonov, Nucl. Instrum. Methods Phys. Res., Sect. B 173, 54 (2001).

[16] H. Bruck, Circular Particle Accelerators (Press Universities of France, Saclay, 1966).

[17] A. A. Kolomensky and A. N. Lebedev, Theory of Cyclic Accelerators (North-Holland, Amsterdam, 1966).

[18] V. V. Anashin et al., Nucl. Instrum. Methods Phys. Res., Sect. A 282, 369 (1989).

[19] H. Hama, S. Takano, and G. Isoyama, Control of Bunch Length on the UVSOR Storage Ring, Workshop on Foth Generation Light Sources, edited by M. Cornacchia and H. Winick, 1992, SSRL 92/02, USA, p. 208.

[20] D. F. Alferov, Yu. A. Bashmakov, and E. G. Bessonov, Sov. Phys. Tech. Phys. 18, 1337 (1974).

[21] D. F. Alferov, Yu. A. Bashmakov, K. A. Belovintsev, E. G. Bessonov, and P. A. Cherenkov, Part. Accel. 9, 223 (1979).

[22] E. G. Bessonov, Proceedings of the Lebedev Physics Institute, Series 214, edited by N. G. Basov and P. A. Cherenkov (Nauka, Moscow, 1993), pp. 3-119 (in Russian).

[23] Y.R. Shen, The Principles of Nonlinear Optics (Wiley, New York, 1984). 
[24] G. Cerullo, and S. De Silvestri, Rev. Sci. Instrum. 74, 1 (2003).

[25] J. A. Armstrong, N. Bloembergen, J. Ducuing, and P. S. Pershan, Phys. Rev. 127, 1918 (1962).

[26] A. Maitland and M.H. Dunn, Laser Physics (NorthHolland, Amsterdam, 1969).

[27] I. N. Ross et al., Opt. Commun. 144, 125 (1997).

[28] K. W. Chang, A. C. Chiang, T. C. Lin, B. C. Wong, Y.H.
Chen, and Y. C. Huang, Opt. Commun. 203, 163 (2002).

[29] L. E. Myers, G. D. Miller, R. C. Eckardt, M. M. Fejer, R. L. Byer, and W. R. Bosenberg, Opt. Lett. 20, 52 (1995).

[30] C. W. Hoyt, M. Sheik-Bahae, and M. Ebrahimzadeh, Opt. Lett. 27, 1543 (2002).

[31] K. Osvay, G. Kurdi, J. Klebniczki, M. Csatari, and I. N. Ross, Appl. Phys. Lett. 80, 1704 (2002). 